\title{
Pulse volume recording for peripheral vascular disease diagnosis in diabetes patients
}

This article was published in the following Dove Press journal:

Journal of Vascular Diagnostics

2I October 2015

Number of times this article has been viewed

\author{
Erik Benitez \\ Bauer E Sumpio \\ Department of Surgery (Vascular), \\ Yale University School of Medicine, \\ New Haven, CT, USA
}

Correspondence: Bauer E Sumpio Department of Surgery (Vascular), Yale University School of Medicine, 333 Cedar Street, New Haven, CT 065 I0, USA

Tel +l 2037856217

Email bauer.sumpio@yale.edu
Abstract: Peripheral arterial disease is common in patients with diabetes mellitus. It has become routine to screen for peripheral arterial disease in diabetic patients. As clinicians' knowledge and understanding of the disease processes increase, the diagnosis and surveillance also rises. As an adjunct to clinical examination, pulse volume recordings are useful in assessing perfusion of patients with poorly compressible arteries. The development of this study during the early 70 s helped create the concept of noninvasive vascular imaging and testing. The analysis of pressure-pulse waveforms localized the presence of high-grade lesions and delineated the extent of the disease process. Pulse volume recordings are currently extensively used with other modalities such as arterial duplex and angiography and help to establish an arterial etiology of a patient's disease process, as well as localizing the lesion, determining the prognosis, and performing surveillance after interventions.

Keywords: pulse volume recordings, ankle brachial index, peripheral arterial disease, diabetes mellitus, critical limb ischemia, pulse wave contour, pressure-pulse waveforms

\section{Introduction}

Patients with diabetes mellitus (DM) are more likely to develop severe forms of peripheral arterial disease (PAD) at an earlier age compared with their nondiabetic counterparts. ${ }^{1}$ Diabetes affects nearly every vascular bed as the invasive nature of diabetes creates a proatherogenic state, which increases the presence of inflammation and derangements in vascular structures, leading to permanent changes in the state of arterial structure and function. ${ }^{2}$ The abnormalities of endothelial function in diabetic patients allow for increased development of atherosclerosis with increase in proinflammatory state: increased leukocyte chemotaxis, adhesion, transmigration, and transformation into foam cells - the earliest precursor of atheroma formation. Additionally, the loss of the normal regulatory function of nitrous oxide as a potent vasodilator to limit inflammation via its modulation of leukocyte-vascular wall interaction leads to a consequence of atherosclerosis and calcification: noncompressible arteries. ${ }^{2}$ Traditionally, the vascular specialist would combine clinical exam along with physiological measurements such as measuring the blood pressure in the extremities and calculating an ankle brachial index (ABI). However, in patients with noncompressible arteries, as in those with DM, the ABI is erroneous or not obtainable. Assessment of the pressurepulse of the arteries in the lower limbs is an important tool for evaluating patients with PAD, as a decrease in amplitude of the pressure-pulse indicates that the lumen of the artery has narrowed. Pulse volume recording (PVR) is a simple, noninvasive screening test developed by Raines et $\mathrm{al}^{3}$ in 1973 , while Raines was still a student researcher at 
Massachusetts Institute of Technology. Initially, similar to the early plethysmograph, the device created by Raines et al incorporated advances in transducer and electronic technology that allowed improved sensitivity in detecting arterial pressure-pulse change as well as creating a recorded trace that was calibrated in pressure and not volume. ${ }^{3}$

\section{History}

During the time of its development, the PVR was evaluated in the newly created vascular laboratories, at the Massachusetts General Hospital in 1972 by Darling et al. ${ }^{4}$ The laboratory combined various noninvasive techniques and instrumentation to diagnose and monitor vascular disease. This diagnostic laboratory was so successful that in the resultant 6 years that followed, 400 similar laboratories opened throughout the country, in both large university medical centers and small community hospital settings. The PVR was eventually used for monitoring of pulse amplitudes both before and after surgery as a way to evaluate the success of revascularization surgery. The early PVR consisted of two, nonoverlapping, air-filled cuffs - one for pressure monitoring and the other for occluding the artery of interest.

To understand the cuff dynamics, one must recognize that compression of air in the cuff by the passage of the arterial pulse is regarded as isentropic due to the negligible heat transfer at the frequencies tested, $0.5-8 \mathrm{~Hz}$. Raines et $\mathrm{al}^{3}$ calculated the small volume deviations in the cuff

$$
\begin{aligned}
& P_{c} V_{c}^{\gamma}=\text { Const } \\
& d V_{c}=k d V_{a}=k C d P_{a} \\
& d P_{c}=\gamma k P_{c}^{2} d P_{a} P_{0}\left(V_{1}+V_{1}+V_{2}\right)-V_{2} P_{c}
\end{aligned}
$$

where $V_{\mathrm{C}}$ is the sum of volumes of air in the cuff and the circuit, $P_{\mathrm{C}}$ denotes the absolute pressure, $\gamma$ is the adiabatic constant (for air, $\gamma=1.4$ ) (Equation 1). The assumption was that the volume changes in the cuff caused by the passage of the pulse were proportional to the changes in the arterial volume, $\mathrm{d} V_{\mathrm{a}}$, encompassed by the cuff(Equation 2). $C=\mathrm{d} V_{\mathrm{a}} /$ $\mathrm{d} P$ a is the compliance of the arterial section surrounded by the cuff and $P_{\mathrm{a}}$ denotes the arterial pressure. If the factor $k C$ remains approximately constant, the variations in the cuff pressure are proportional to the variations in the arterial pressure, and the output of the pressure-sensitive transistor will yield the true arterial pulse contour (Equation 3 ). ${ }^{3}$

To obtain the closest reading to arterial pulse-pressure, Raines et $\mathrm{al}^{3}$ compared readings taken at the brachial artery with the pressure cuff inflated at 30 and $100 \mathrm{mmHg}$
(Figure 1). The tracings corresponding to intermediate pressure cuff values were found between these two limiting curves. He demonstrated that the lower the cuff pressure, the closer the PVR is to the true pressure. The cuff dampens the transmural pressure in the artery beneath it, which at higher pressure levels, such as $100 \mathrm{mmHg}$, produced a marked distortion in the pulse contour that is greatest during the diastolic phase of the pulse. The increase in the change of $V_{\mathrm{C}}$ with cuff pressure can be explained in part by the dependence of arterial compliance on transmural pressure. Raines et $\mathrm{al}^{3}$ observed that arterial compliance increased with lowering transmural pressures, thereby decreasing the volume excursion of the selected artery and vice-versa. Darling et $\mathrm{al}^{4}$ also tested the frequency response of the device by strapping a water-filled bladder around a rigid plastic cylinder and then encircled it with an air-filled PVR monitoring cuff. A pistonin-cylinder pump was then connected to the bladder so as to produce a sinusoidal pressure change in the monitoring cuff. The amplitude of these oscillations remained constant up to a pump frequency of $20 \mathrm{~Hz}$, which approximates the higher frequencies of the human arterial pulse contour.

When evaluating pressure-pulse waveforms, under normal physiological conditions, the reflection of waves in the periphery are mostly dampened out by three factors: the branching pattern, capacitance, and friction inherent in the arterial vasculature. ${ }^{5}$ Reflected waves and secondary humps in the peripheral pulse can be observed due to the occurrence of reflection within the arterial tree. The distal boundary condition that dampens and reduces this reflected wave likely occurs in the transitional regions, as large arteries become small arteries and then small arteries become arterioles.

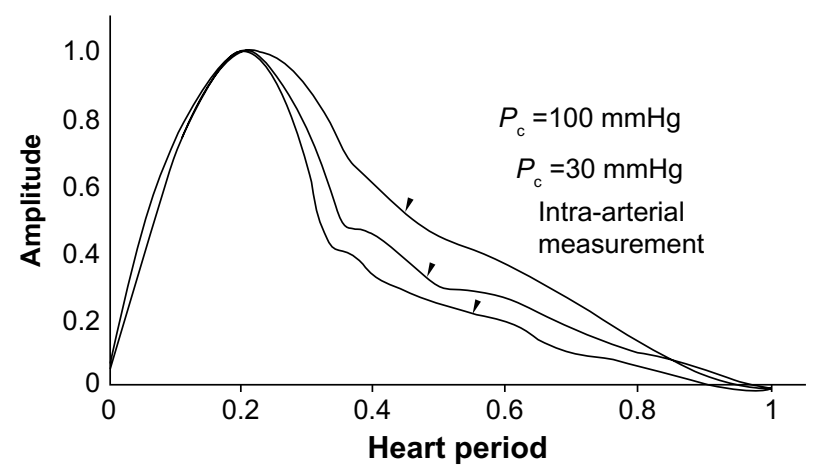

Figure I Comparison of pressure contours obtained with the PVR and direct cannulation of the common femoral artery.

Notes: Amplitude 1.0 = a total of $20 \mathrm{~mm}$ of chart deflection. Heart period measured in seconds. Copyright (C) 1973. Figure reprinted with permission. Originally published in Med Instrum. 1973;7(4):245-250. Raines JK, Jaffrin MY, Rao S. A noninvasive pressure-pulse recorder: development and rationale. Any other usage or printing of AAMI-copyrighted material requires written permission from AAMI. ${ }^{3}$ Abbreviations: $P_{\mathrm{C}}$, absolute pressure; PVR, pulse volume recording. 
Resistance to flow progressively increases as lumen diameter progressively decreases working in combination with the vessel compliance to function as an acoustic muffler to diminish the propagation of reflected waves. Raines et $\mathrm{al}^{5}$ proposed a model of complex load impedance, representing the vascular bed

$$
Z_{T}=R_{1}+\frac{R_{2}\left(1-j R_{2} C_{T} W\right)}{1+R_{2}^{2} C_{T}^{2} W_{2}^{2}}
$$

where the sum resistances $\left(Z_{T}\right)\left(R_{1}\right.$ and $R_{2}$ represent lumped compliances) is equal to the terminal resistance. $C_{T}$ is the lumped compliances of the vessel representing distensibility of the small vessels.

Figure 2 demonstrates the physiological model of the pressure-pulse waveforms of the major arteries of a young, healthy adult leg beginning at the iliac bifurcation and ending in the popliteal vessels, prior to its trifurcation into tibial vessels. Raines et $\mathrm{al}^{5}$ performed angiographic studies on 24 adults documenting vessel length, lumen diameters, as well as pulse wave velocity (PWV). The concept of PWV, a measure of arterial stiffness, was introduced in "Functions of the Heart and Arteries" (1808) by English physiologist Thomas Young prior to his training in becoming a physician. ${ }^{6}$ The findings in Raines et $\mathrm{al}^{5}$ study are consistent with those of Cachovan et al, ${ }^{7}$ who evaluated the PWV of 40 men with segmental impedance plethysmography, in which the medical examiners were unable to detect significant variation in PWV in areas where atherosclerotic disease was not present. Postmortem

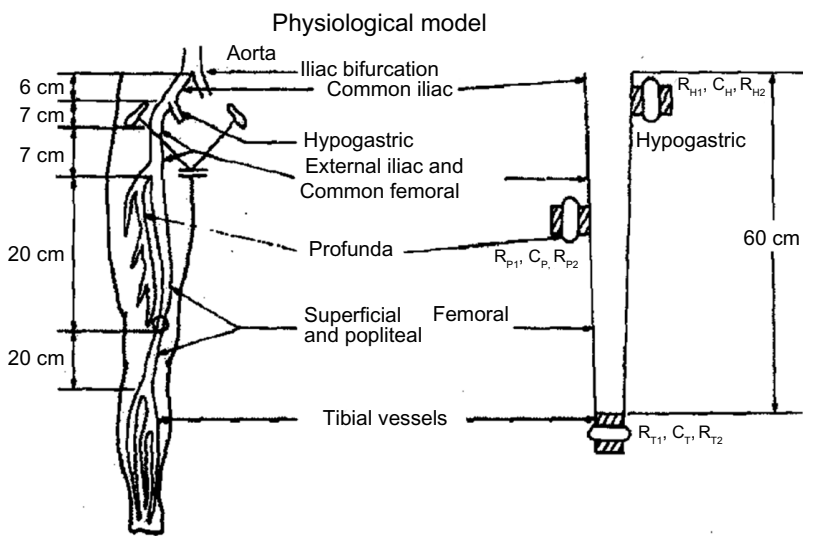

Figure 2 The physiological model showing the anatomical sketch on the left and a schematic of the simulated arteries on the right.

Notes: "C" represents the lumped/total value of compliance in that area for that vessel. The subscript letters denote the vessel each value corresponds to: $\mathrm{H}=$ hypogastric; $\mathrm{T}=$ tibial, and $\mathrm{P}=$ profunda; with the exception of. "R" represents the value of resistance for that vessel. Subscript , indicates the occluding cuff, and subscript ${ }_{2}$ indicates the peripheral resistance of the extremity at that area. Reprinted from the Journal of Biomechanics; 7(I); Raines JK, Jaffrin MY, Shapiro AH; A computer simulation of arterial dynamics in the human leg; 77-91; Copyright (C) 1974, with permission from Elsevier. ${ }^{5}$ studies were performed, which suggested that if the PWV was greater than $1,200 \mathrm{~cm} / \mathrm{s}$ in normotensive patients, there was high probability of disease throughout the vasculature of the lower extremities. Zangenek and Nassereslami ${ }^{8}$ similarly compared PWV between foot-to-foot in 640 test subjects without significant PAD and concluded that: 1) PWV varies little with aging in the absence of PAD, 2) PWV can be increased by $100 \%$ in the presence of hypertension, and 3) patients with a mean average of $100 \mathrm{mmHg}$ for systolic blood pressure had PWV of 1,037 $186 \mathrm{~cm} / \mathrm{s}$ which was similar to the early studies. ${ }^{7,9}$

Guha $^{10}$ reported that the systolic and reflected waves amplify as the pulse travels away from the iliac vessels, during which the mean pressure falls gradually due to friction and the mean flow decreases from branching in the arterial tree (Figure 3). The PWV between the iliac and tibial vessels was calculated to be $1,279 \mathrm{~cm} / \mathrm{s}$, while the PWV below the tibial vessels decreases significantly. This difference in PWV can be attributed to the fact that waves travel faster in a higher-pressure system.

Arteriosclerosis thickens the vessel tissue and causes a loss of compliance in the arterial wall. A change in compliance changes the strength of the distributed reflections as well as the reflection coefficient at the distal end of the vasculature model. ${ }^{4}$ The abnormal pulse contours observed in patients with arteriosclerosis can be reproduced in the model if the reflection coefficient is large and negative. When the peripheral resistances are increased while impedance is constant, the reflection waves increase as the peaks of the pulse heighten and the valleys deepen. Raines et $\mathrm{al}^{3}$ tested this by utilizing a monitoring cuff placed proximally on the leg that recorded pulse volumes, while the distal cuff was insufflated with varying values (Figure 4). They demonstrated that as the cuff partially occluded the arteries at the distal site, the reflected waves decreased in amplitude, but when the cuff completely occluded the arteries, the reflected waves then increased as a stiffer reflection site was produced. They concluded that vasoconstriction increases peripheral resistance and increases the magnitude of the reflected waves, while vasodilation decreases the peripheral resistance and diminishes the reflected wave.

Another innovation pioneered by O'Donnell et $\mathrm{al}^{11}$ for PVR was its use for intraoperative monitoring during lower extremity bypass procedure. In the procedure, simulated pulse saline solution flow is monitored by PVR to obtain physiological information on distal runoff site prior to and after the anastomoses to examine for successful revascularization vs early obstruction (Figure 5). A successful bypass graft results 


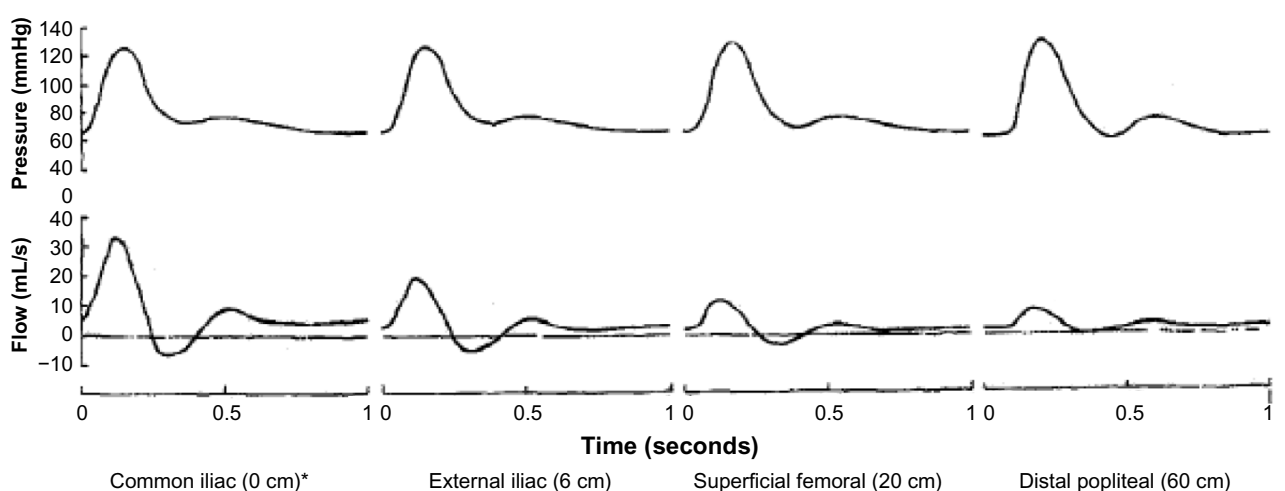

Figure 3 Propagation of pressure and flow waveforms along the main artery.

Notes: *Length from iliac bifurcation. Reprinted from the Journal of Biomechanics; 7(I); Raines JK, Jaffrin MY, Shapiro AH; A computer simulation of arterial dynamics in the human leg; 77-91; Copyright (C) 1974, with permission from Elsevier. ${ }^{5}$

A

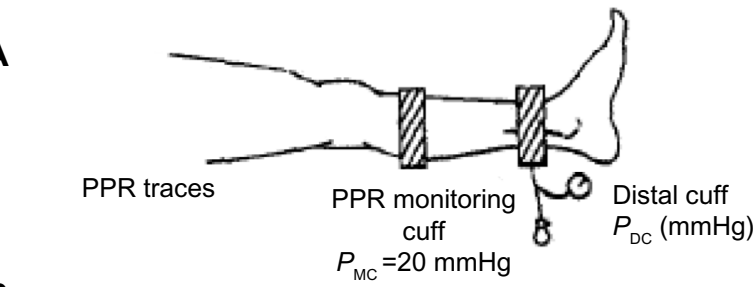

B
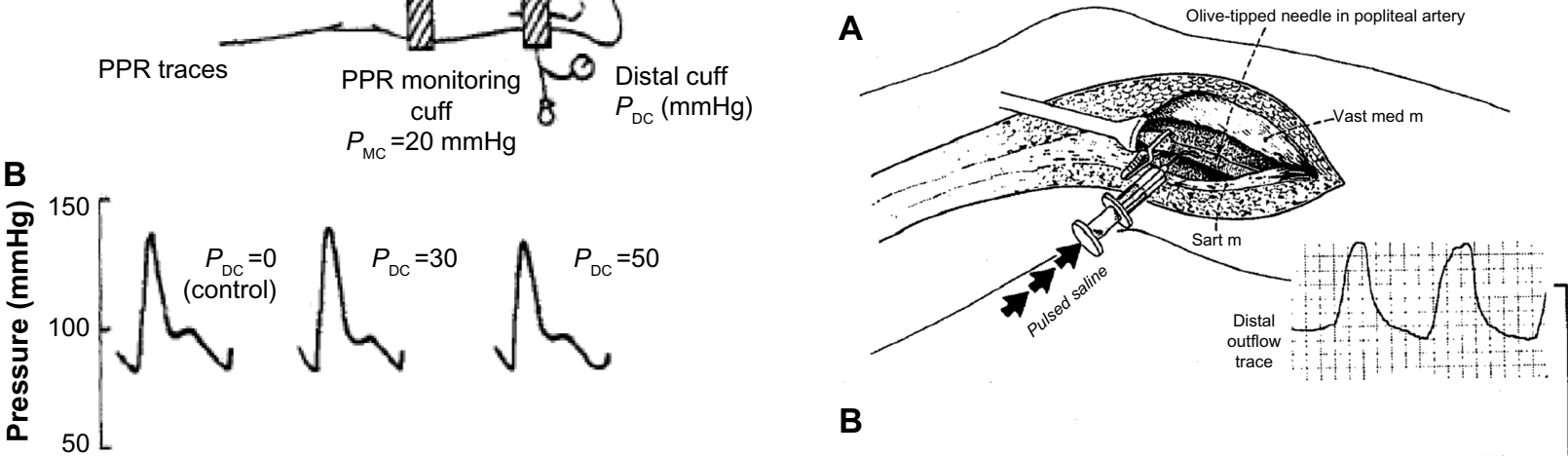

B
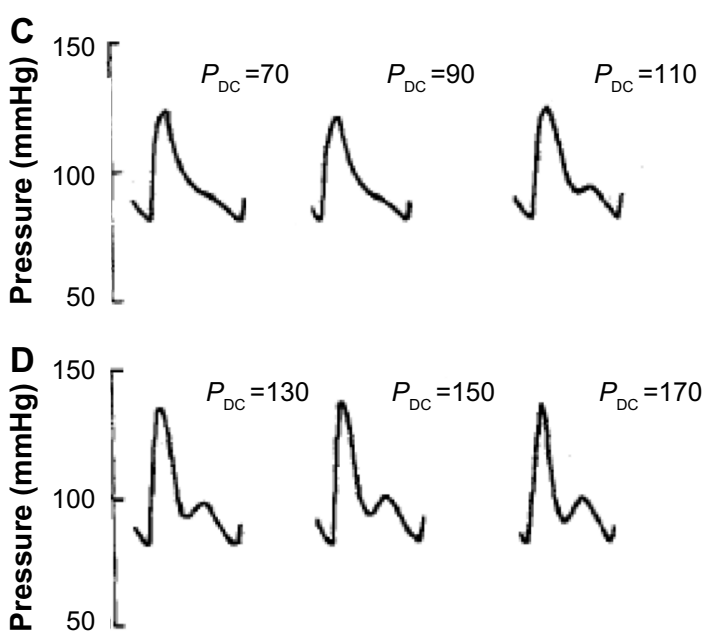

Figure 4 Effect of a distal cuff on the pressure-pulse in the calf.

Notes: (A) Resistance applied distal to PPR recording. The monitoring cuff is set at a mean pressure of $20 \mathrm{~mm} \mathrm{Hg}$. The distal cuff shows the pressure at which wave reflection is prominent. Figures B-D show that as the distal cuff pressure increases, the distal resistance increases which enhances the wave reflection. (B) As the arteries begin to partially occlude, the reflected wave begins to decrease in amplitude. (C) The maximum decrease in wave amplitude is achieved with significant effect on the wave morphology. (D) The artery here is completely occluded which produces a stiffer reaction at the ankle and returns the waveform to its original amplitude. Reprinted from the Journal of Biomechanics; 7(I); Raines JK, Jaffrin MY, Shapiro AH;A computer simulation of arterial dynamics in the human leg; 77-91; Copyright (C) 1974, with permission from Elsevier. ${ }^{5}$ Abbreviations: $\mathrm{P}_{M C}$, pressure monitoring cuff; $\mathrm{P}_{D C}$, pressure distal cuff; PPR, pressure pulse recorder.

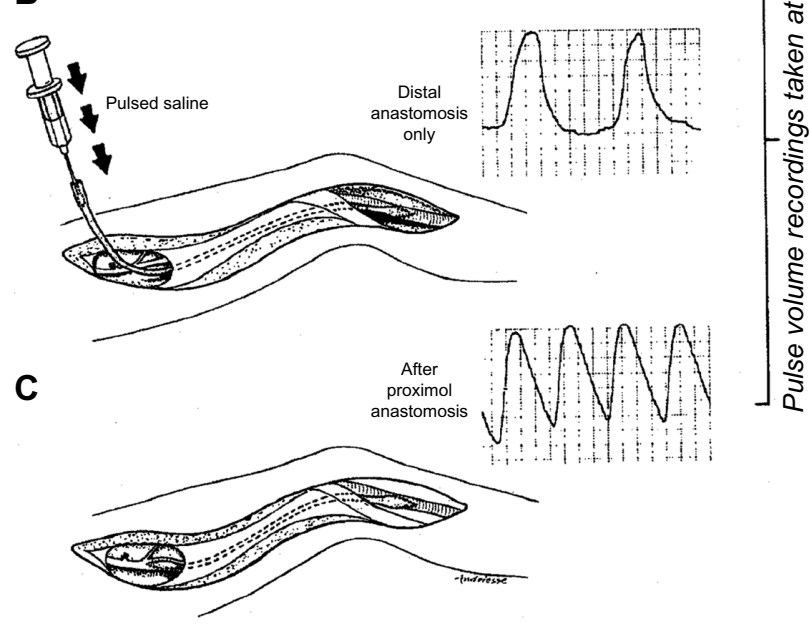

Figure 5 The technique of intraoperative monitoring utilizing the PVR with simulated pulsed saline solution flow provides physiological information on the distal runoff prior to and after creation of a bypass anastomoses.

Notes: The PVR used in conjunction with the simulated pulsed saline to solution flow provides physiological information on the distal runoff prior to insertion of the graft, and the condition and function of the distal and proximal anastomosis. (A) The olive tipped needle is inserted into the popliteal artery at the site of the distal arteriotomy. (B) A heparinized saline solution is pulsed through the upper end of the saphenous vein. (C) After completion of the femoral anastomosis, a final PVR tracing is obtained. Reprinted with permission from the Journal of the American College of Surgeons, formerly Surgery Gynecology \& Obstetrics. Copyright () 1977. Reprinted from: O'Donnell TF Jr, Raines JK, Darling RC. Intraoperative monitoring using the pulse volume recorder. Surg Gynecol Obstet. 1977; |45(2):252-254..$^{10}$

Abbreviations: PVR, pulse volume recording; Sart m, sartorius muscle; Vast med $\mathrm{m}$, vastus medialis muscle. 
in an increase in the amplitude and a change in the contour of the PVR tracing. Failure to detect any change was considered indicative of a technical error and mandated immediate re-evaluation of the distal anastomosis (the frequent site of technical failure). This timely prospective detection of a technical error allowed quick intervention, preventing unnecessary limb loss and need for repeat operative interventions.

\section{Clinical applications}

The clinical presentation of symptomatic PAD patient usually involves ulceration, claudication, or ischemic pain. However, in the presence of DM, patients often present with more atypical symptoms such as leg fatigue, recurrent stenosis or occlusion of tibial vessels, and peripheral neuropathy, masking the pain associated with this severe disease process. Additionally, DM patients with PAD are at increased risk for acute thrombosis complicated by major amputation. ${ }^{12}$ This is also due to poor collateral blood flow as DM targets the microvasculature that leads to an incomplete pedal arch resulting in more severe and acute ischemic conditions in the lower extremities. ${ }^{13}$ Patients with DM frequently have calcification of the tunica media of blood vessels as well as noncompressibility due to medial sclerosis of arteries. These qualities of the disease process in DM cause lower extremity blood pressure measurement such as ABI (Table 1) to be inaccurate for diagnosis and surveillance as they are often falsely elevated $>1.3$ due to incompressibility of vessels. ${ }^{14}$

The inconsistent compressibility of arteries of patients with DM has led to the search for other modalities to use

Table I Classification of $A B \mid$ scores

\begin{tabular}{ll}
\hline ABI & Interpretation \\
\hline$>1.3$ & False elevation; heavy vessel calcification \\
I.0-I.29 & Normal \\
$0.91-1.0$ & Borderline \\
$0.7-0.9$ & Mild PAD \\
$0.4-0.69$ & Moderate PAD \\
$<0.4$ & Severe PAD, associated critical limb \\
& ischemia (ulceration and rest pain) \\
\hline
\end{tabular}

Notes: A normal or subnormal $A B I$ in a diabetic patient should prompt health care providers to consider the possibility of even worse vascular disease than the value would imply in non-diabetic patients. Additionally a falsely elevated $A B I$, such as greater than I.3, which is common in DM patients, is associated with increased cardiovascular event rates. $A B I$ is derived first by determining the systolic blood pressure in both brachial arteries and then in the dorsalis pedis and posterior tibial arteries. It can then be calculated for each lower extremity and interpreted as shown in the table. To determine the $A B I$ score, after measuring the brachial systolic value one must evaluate the systolic values of the lower extremities. Adapted from Clinics in Podiatric Medicine and Surgery; 3I(I); Chin JA, Sumpio BE; Diabetes mellitus and peripheral vascular disease: diagnosis and management; II-26; Copyright (C) 2014, with permission from Elsevier.'

Abbreviations: DM, diabetes mellitus; $A B I$, ankle brachial index; PAD, peripheral arterial disease.
Table 2 PVR is classified into five categories that are combined with pressure data to define states of vascular disease

\begin{tabular}{lll}
\hline PVR & \multicolumn{2}{l}{ Chart deflection $(\mathbf{m m})$} \\
\cline { 2 - 3 } category & Thigh and ankle & Calf \\
\hline 1 & $>15^{\mathrm{a}}$ & $>20^{\mathrm{a}}$ \\
2 & $>15^{\mathrm{b}}$ & $>20^{\mathrm{b}}$ \\
3 & $5-15$ & $5-20$ \\
4 & $<5$ & $<5$ \\
5 & Flat & Flat \\
\hline
\end{tabular}

Notes: ${ }^{a}$ Without reflected wave; ${ }^{b}$ with reflected wave. Reprinted with permission from Elsevier. This table was published in Medical Clinics of North America, 64(2), Raines J, Traad E, Noninvasive evaluation of peripheral vascular disease, 283-304, Copyright (C) Elsevier 1980.20

Abbreviations: PVR, pulse volume recording; mm, millimeter.

in the evaluation of PAD in those patients. As discussed, PVR introduced by Darling et al almost 35 years ago appears to be suited for this process. ${ }^{4}$ PVR is now currently used as a complementary noninvasive vascular study with ABI or plethysmography to evaluate physiological markers in the lower extremities. Due to the artificially elevated ankle pressures secondary to incompressibility of blood vessels in DM patients, PVR tracings are one of a few reliable and measurable parameters. The technique in a modern vascular lab is not too different from the model developed by Darling et $\mathrm{al}^{4}$ and utilizes multiple pneumatic cuffs and pressure sensors at different levels on each leg. PVR interpretation

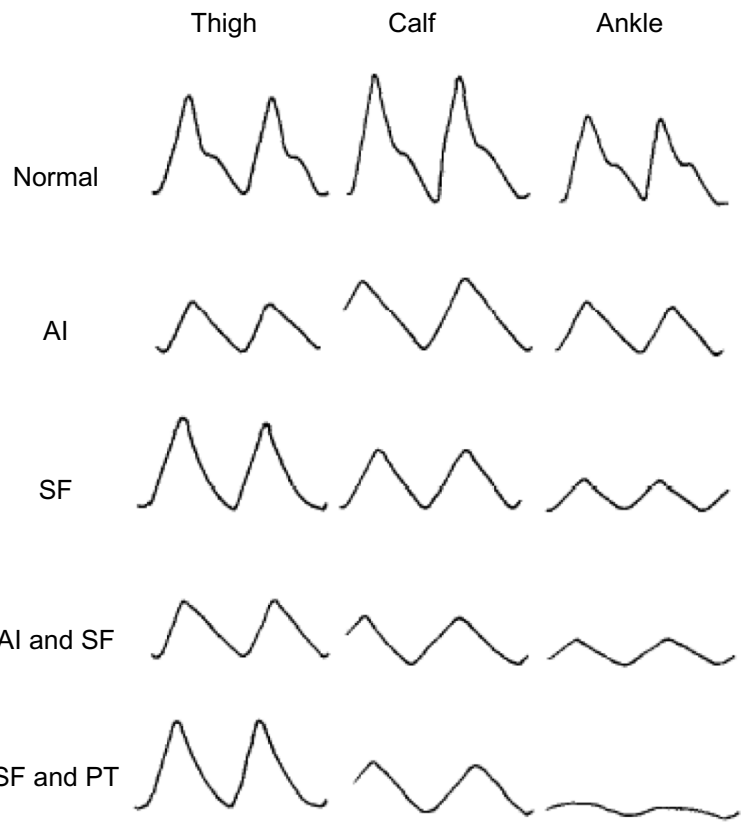

Figure 6 PVR of normal patients alongside PAD patients with various combinations of localized lesions.

Notes: Reprinted from The American Journal of Surgery; 138(2); Rutherford RB, Lowenstein DH, Klein MF; Combining segmental systolic pressures and plethysmography to diagnose arterial occlusive disease of the legs; 2II-2 I8; Copyright (C) 1979, with permission from Elsevier. ${ }^{16}$

Abbreviations: Al, aortoilliac; PAD, peripheral arterial disease; PT, posterior tibial; PVR, pulse volume recording; SF, superficial femoral. 


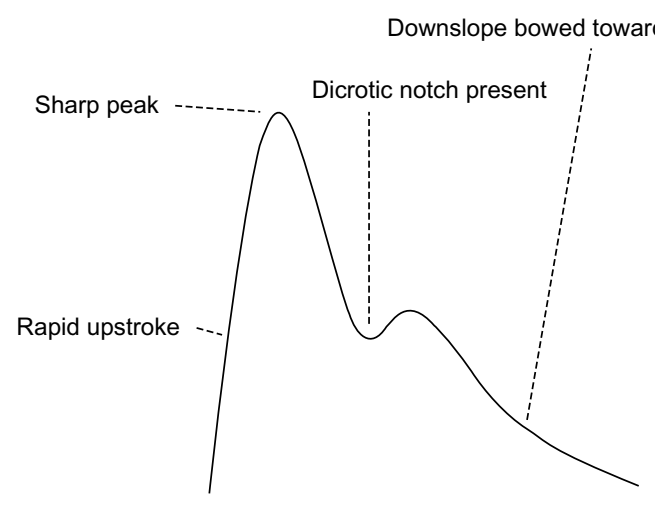

Normal tracing

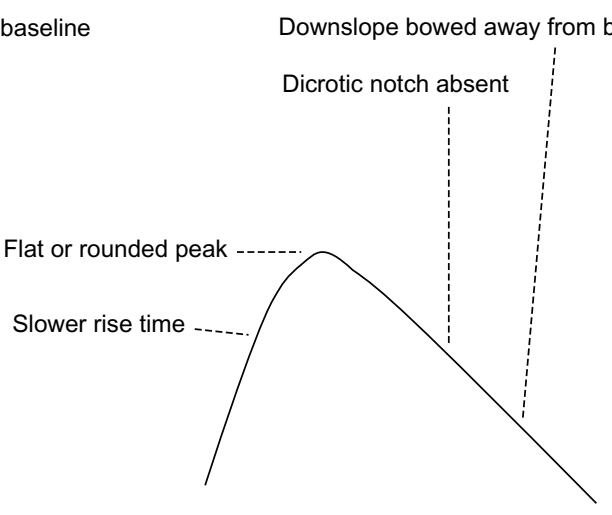

Abnormal tracing

Figure 7 The differences between a normal and abnormal PVR tracing.

Notes: A normal tracing is characterized by a rapid upstroke, sharp peak, presence of a dicrotic notch, and a downslope that is bowed toward the baseline. An abnormal tracing demonstrates a slower rise time, a flat or rounded peak, absence of the dicrotic notch, and a downslope that is bowed away from the baseline. Reprinted from Radiologic Clinics of North America; 52(6); McCann TE, Scoutt LM, Gunabushanam G; A practical approach to interpreting lower extremity noninvasive physiologic studies; I343-1357; Copyright $@ 2014$, with permission from Elsevier. ${ }^{14}$

Abbreviation: PVR, pulse volume recording.

involves analyzing both the amplitude and contour tracings to recognize specific patterns to identify areas of stenosis in the affected extremity (Figure 5). Tracings show the changes of volume within an extremity during arterial pulsation. By standardizing the volume of air and pressure within the cuff based on the seminal work of Darling et al, ${ }^{4}$ the subtle volume changes that occur in a limb lead to measurable pulsatile pressure changes within the cuff that reflect changes in arterial pressure. Chart deflections for PVR tracings and limb pressures decrease with worsening vascular disease (Tables 1 and 2). These variations in the contour and amplitude of the PVR tracings provide a qualitative assessment of the degree of PAD (Figure 6).

The normal pulse wave is characterized by a steep upslope, narrow peak, and the presence of a dicrotic notch in the down slope. Pulse wave contour and amplitude should be symmetric when comparing both limbs at the same level. There is usually a loss of the dicrotic notch in the presence of a proximal arterial stenosis or occlusion (Figure 7). The amplitude of the pulse wave diminishes progressively in the presence of worsening atherosclerotic disease. As PAD worsens, there is a dampening of the contour, while the peak appears broader and more rounded, and the down slope becomes convex away from the baseline. ${ }^{15}$ Eventually the contour will flat line, reflecting either severe PAD or critical limb ischemia. Rutherford et a ${ }^{16}$ evaluated 217 limbs with segmental limb pressures, PVR, and angiography. Their findings of PVR measured at the thigh, calf, and ankle confirmed the previously published results by Darling et $\mathrm{al}^{4}$ on its clinical utility. By comparing segmental pressures and PVR to angiography, the physician interpretation has a diagnostic accuracy of $86 \%$. When used in addition to traditional angiography, the diagnostic accuracy improves to $95 \%$.

Lewis and Owens ${ }^{17}$ performed a cross-sectional study of degree of PAD in bilateral lower extremities of 205 diabetic subjects through serial evaluation of their pedal pulses, ABI, Doppler waveforms, and PVR. They found that PVR when compared with the color spectral waveforms had a high level of correlation at $91.7 \%(n=189)$. The other noninvasive combinations correlated less than $60 \%$. This indicated that PVR should be considered for preliminary noninvasive vascular assessment, as it is easy to use and would reduce the number of inappropriate referrals while providing meaningful results for the vascular specialist. In a related study, Moneta et al ${ }^{18}$ evaluated the effectiveness of segmental pulse pressure and arterial duplex in locating significant stenotic or occlusive lesions in the lower extremities. They evaluated 151 lower extremities with angiography for patients with ischemic rest

Table 3 Summary of data that provides objective criteria for evaluating PAD patients with complaints of ischemic rest pain

\begin{tabular}{llll}
\hline & Unlikely & Probable & Likely \\
\hline Ankle pressure $(\mathrm{mmHg})$ & & & \\
$\quad$ Nondiabetic & $>55$ & $35-55$ & $<35$ \\
$\quad$ Diabetic & $>80$ & $55-80$ & $<35$ \\
Ankle PVR category & & & \\
$\quad$ Nondiabetic and diabetic & $\mathrm{I}, 2,3$ & 3,4 & 4,5 \\
\hline
\end{tabular}

Notes: Ankle PVR categories refer to amount of decreased chart deflection $(\mathrm{mm})$ caused by PAD that alters the tracing displayed from a normal and healthy tracing. At a maximum of 5 , there is severe disease as there is no waveform and minimal chart deflection is present. Reprinted with permission from Elsevier. This table was published in Medical Clinics of North America, 64(2), Raines J, Traad E, Noninvasive evaluation of peripheral vascular disease, 283-304, Copyright ( Elsevier 1980.20

Abbreviations: PAD, peripheral arterial disease; PVR, pulse volume recording; $\mathrm{mmHg}$, millimeter of mercury. 
pain, gangrene, or claudication (Table 3). These limbs were then evaluated with either segmental pulse pressure or arterial duplex and compared against angiography. They demonstrated that arterial duplex was superior to segmental pulse pressures for identifying high-grade lesions in the iliofemoropopliteal region. Segmental pulse pressures had a higher level of false positives. Overall, the agreement between angiography and arterial duplex in locating hemodynamically significant lesions was $82 \%$ of the limbs studied. However, the correlation between segmental Doppler pressures and angiography was not as strong, occurring in only $34 \%$ of the limbs. ${ }^{18}$ By comparing segmental pressures and PVR to angiography, physician interpretation has a diagnostic accuracy of $86 \%$. When PVR is used in addition to traditional angiography, the diagnostic accuracy improves to $95 \% .{ }^{16}$ This system allows for anatomical localization of hemodynamically significant lesions in the vasculature of the lower extremities.

The physician must be aware of the presence of aortic valvular stenosis, hypotension, tachycardia, or significant proximal arterial stenosis that may mask the presence of distal disease due to decreased intra-arterial pressure. The aforementioned factors diminish the sensitivity of PVR in detecting lesions.

\section{Conclusion}

Noninvasive vascular laboratory physiologic studies are used in screening asymptomatic individuals with risk factors for PAD, helping to establish an arterial etiology of a patient's symptoms, localizing the level of disease, determining the prognosis, and performing surveillance after invasive therapy. ${ }^{19}$ The PVR has since become an indispensable tool in the noninvasive vascular laboratory for obtaining information regarding a patient's perfusion in the lower extremities.

\section{Disclosure}

The authors report no conflicts of interest in this work.

\section{References}

1. Chin JA, Sumpio BE. Diabetes mellitus and peripheral vascular disease: diagnosis and management. Clin Podiatr Med Surg. 2014;31(1): $11-26$.

Journal of Vascular Diagnostics

\section{Publish your work in this journal}

Journal of Vascular Diagnostics is an international, peer-reviewed journal of diagnostics, focusing on non invasive vascular investigation methods involved in the evaluation of vascular diseases. The journal is committed to the rapid publication in the fields of vascular diseases. Original research, review, case reports, expert opinion and
2. American Diabetes Association. Peripheral arterial disease in people with diabetes. Diabetes Care. 2003;26(12):3333-3341.

3. Raines JK, Jaffrin MY, Rao S. A noninvasive pressure-pulse recorder: development and rationale. Med Instrum. 1973;7(4):245-250.

4. Darling RC, Raines JK, Brener BJ, Austen WG. Quantitative segmental pulse volume recorder: a clinical tool. Surgery. 1972;72(6):873-877.

5. Raines JK, Jaffrin MY, Shapiro AH. A computer simulation of arterial dynamics in the human leg. J Biomech. 1974;7(1):77-91.

6. Young T. The croonian lectures: on the functions of the heart and arteries. Phil Trans Roy Soc Lond. 1808;99:1-31.

7. Cachovan M, Dejdar R, Roubkova H, Linhart J, Prerovsky I. Changes of some parameters of arterial elasticity in man during early atherosclerosis of the lower limbs. Angiology. 1969;20(9):520-525.

8. Zangenek VM, Nassereslami H. Das Verbalten der Pulswellengeschwendihert ins Bein in Abhamgigkeit von Lebensalter und Geschlecht. [The behavior of pulse wave velocity in the leg depending on age and sex]. Z Kreislanfforschung. 1966;56(4). German

9. Mozersky DJ, Sumnfr DS, Hokanson DE, Strandness JR DE. Transcutaneous measurement of the elastic properties of the human femoral artery. Circulation. 1972;46(5):948-955.

10. Guha SK. Haemodynamics of the small arterial region in the femoral vascular bed. Med Biol Eng. 1970;8(3):291-299.

11. O’Donnell TF Jr, Raines JK, Darling RC. Intraoperative monitoring using the pulse volume recorder. Surg Gynecol Obstet. 1977;145(2): 252-254.

12. McDaniel MD, Cronenwett JL. Basic data related to the natural history of intermittent claudication. Ann Vasc Surg. 1989;3(3):273-277.

13. De Vivo S, Palmer-Kazen U, Kalin B, Wahlberg E. Risk factors for poor collateral development in claudication. Vasc Endovasc Surg. 2005;39(6):519-524.

14. Edmonds ME, Morrison N, Laws JW, Watkins PJ. Medial arterial calcification and diabetic neuropathy. $\mathrm{Br}$ Med J. 1982;284(6320): 928-930.

15. McCann TE, Scoutt LM, Gunabushanam G. A practical approach to interpreting lower extremity noninvasive physiologic studies. Radiol Clin North Am. 2014;52(6):1343-1357.

16. Rutherford RB, Lowenstein DH, Klein MF. Combining segmental systolic pressures and plethysmography to diagnose arterial occlusive disease of the legs. Am J Surg. 1979;138(2):211-218.

17. Lewis JE, Owens DR. The pulse volume recorder as a measure of peripheral vascular status in people with diabetes mellitus. Diabetes Technol Ther. 2010;12(1):75-80.

18. Moneta GL, Lee RW, Porter JM. Noninvasive localization of arterial occlusive disease: a comparison of segmental Doppler pressures and arterial duplex mapping. J Vasc Surg. 1993;17(3):578-582.

19. Benitez E, Sumpio BJ, Chin J, Sumpio BE. Contemporary assessment of foot perfusion in patients with critical limb ischemia. Semin Vasc Surg. 2014;27(1):3-15.

20. Raines J, Traad E. Noninvasive evaluation of peripheral vascular disease. Med Clin North Am. 1980;64(2):283-304.

\section{Dovepress}

commentaries are all considered for publication. The manuscript management system is completely online and includes a very quick and fair peer-review system, which is all easy to use. Visit http://www.dovepress. com/testimonials.php to read real quotes from published authors. 